\title{
Light modulated cnidocyte discharge predates the origins of eyes in Cnidaria 10
}

\author{
Natasha Picciani $^{1}$ (D) | Jamie R. Kerlin ${ }^{1}$ (D) | Katia Jindrich ${ }^{2}$ (D) | Nicholai M. Hensley ${ }^{1}$ (D) | \\ David A. Gold ${ }^{3}$ (D) | Todd H. Oakley ${ }^{1}$ (D)
}

${ }^{1}$ Department of Ecology, Evolution and Marine Biology, University of California at Santa Barbara, Santa Barbara, CA, USA

${ }^{2}$ School of Biosciences, Cardiff University, Cardiff, UK

${ }^{3}$ Department of Earth and Planetary Sciences, University of California at Davis, Davis, CA, USA

\section{Correspondence}

Natasha Picciani and Todd H. Oakley, Department of Ecology, Evolution and Marine Biology, University of California at Santa Barbara, Santa Barbara, CA 93106 , USA.

Emails: natasha.picciani@gmail.com (NP); todd.oakley@lifesci.ucsb.edu (TO)

\section{Present address}

Natasha Picciani, Department of Ecology and Evolutionary Biology, Yale University, New Haven, CT, USA

Jamie R. Kerlin, Department of Biology, California State University, Northridge, CA, USA

Funding information

Coordenação de Aperfeiçoamento de Pessoal de Nível Superior, Grant/Award Number: BEX-13130-13/7; Division of Integrative Organismal Systems, Grant/ Award Number: 1754770

\begin{abstract}
Complex biological traits often originate by integrating previously separate parts, but the organismal functions of these precursors are challenging to infer. If we can understand the ancestral functions of these precursors, it could help explain how they persisted and how they facilitated the origins of complex traits. Animal eyes are some of the best studied complex traits, and they include many parts, such as opsin-based photoreceptor cells, pigment cells, and lens cells. Eye evolution is understood through conceptual models that argue these parts gradually came together to support increasingly sophisticated visual functions. Despite the well-accepted logic of these conceptual models, explicit comparative studies to identify organismal functions of eye precursors are lacking. Here, we investigate how precursors functioned before they became part of eyes in Cnidaria, a group formed by sea anemones, corals, and jellyfish. Specifically, we test whether ancestral photoreceptor cells regulated the discharge of cnidocytes, the expensive single-use cells with various functions including prey capture, locomotion, and protection. Similar to a previous study of Hydra, we show an additional four distantly related cnidarian groups discharge significantly more cnidocytes when exposed to dim blue light compared with bright blue light. Our comparative analyses support the hypothesis that the cnidarian ancestor was capable of modulating cnidocyte discharge with light, which we speculate uses an opsin-based phototransduction pathway homologous to that previously described in Hydra. Although eye precursors might have had other functions like regulating timing of spawning, our findings are consistent with the hypothesis that photoreceptor cells which mediate cnidocyte discharge predated eyes, perhaps facilitating the prolific origination of eyes in Cnidaria.
\end{abstract}

\section{KEYWORDS}

light sensing, nematocysts, ocelli, photoreception, photosensitivity 


\section{1 | INTRODUCTION}

Complex biological traits often evolve by combining previously separate parts, which we herein term "precursors," that originally served other organismal functions. Understanding ancestral functions of precursors will help us understand whether and how they were conserved over time, ultimately informing how complex traits originate. An attractive system for exploring the ancestral functions of precursors is animal eyes, which are complex organs composed of modules with known functions, including opsin-based photoreceptors, pigments, and often lens cells (Oakley \& Speiser, 2015). These modules also function outside of eyes, yet only when combined do they facilitate the complex visual tasks that eyes can do. According to a functional model, modules gradually accrued during eye evolution, sequentially adding photoreceptors, pigments, and lenses to support the acquisition of increasingly advanced visual tasks (Nilsson, 2013). The modules did not evolve de novo within eyes but probably were recruited from elsewhere, while also serving functions outside of eyes (Swafford \& Oakley, 2019). As such, understanding the functions of precursor modules that would later join forces and become eyes is particularly important for understanding eye origins.

Photoreceptor cells are a logical starting point for understanding eye origins because they are the keystone module of animal eyes. When they are outside of eyes, photoreceptor cells are called extraocular, lack a visual function, and simply sense the ambient intensity of light (Ramirez et al., 2011). Still, they provide nondirectional information on light levels that is useful to organisms for many sensory tasks, including shadow responses, circadian and seasonal entrainment, depth gauges, and other organismal functions (Nilsson, 2009). From the perspective of the functional model of eye evolution, extraocular photoreceptors predated their incorporation into eyes by functioning as simple light gauges for nondirectional photoreception (Nilsson, 2013). Although generally associated with nondirectional photoreception, the organismal-level functions of eye precursors often go untested.

We propose cnidarians (sea anemones, corals, and jellyfish) are a particularly interesting system for examining possible early functions of eye precursors. Cnidarians convergently evolved eyes of many types in lineages with a jellyfish stage, including lensed eyes with crystallins in box jellyfish (Miranda \& Collins, 2019; Picciani et al., 2018). At the same time, ancestral cnidarians lacked eyes altogether but possessed opsin proteins that may have been multimodal (Leung \& Montell, 2017) and were probably capable of sensing light (Picciani et al. 2018). Therefore, any functions relying on nondirectional light sensing in the cnidarian ancestor may represent an early role of eye precursors. Nondirectional light sensing in Cnidaria is associated with various sensory tasks, including larval settlement and synchronized mass spawning in corals (Boch et al., 2011; Mason et al., 2012), vertical migration and spawning in jellyfish (Miller, 1979; Quiroga Artigas et al., 2018; Schuyler \& Sullivan, 1997), tentacle expansion and retraction in corals and sea anemones (Gorbunov \& Falkowski, 2002; Sawyer et al., 1994), and cnidocyte discharge in Hydra polyps (Plachetzki et al., 2012). Among

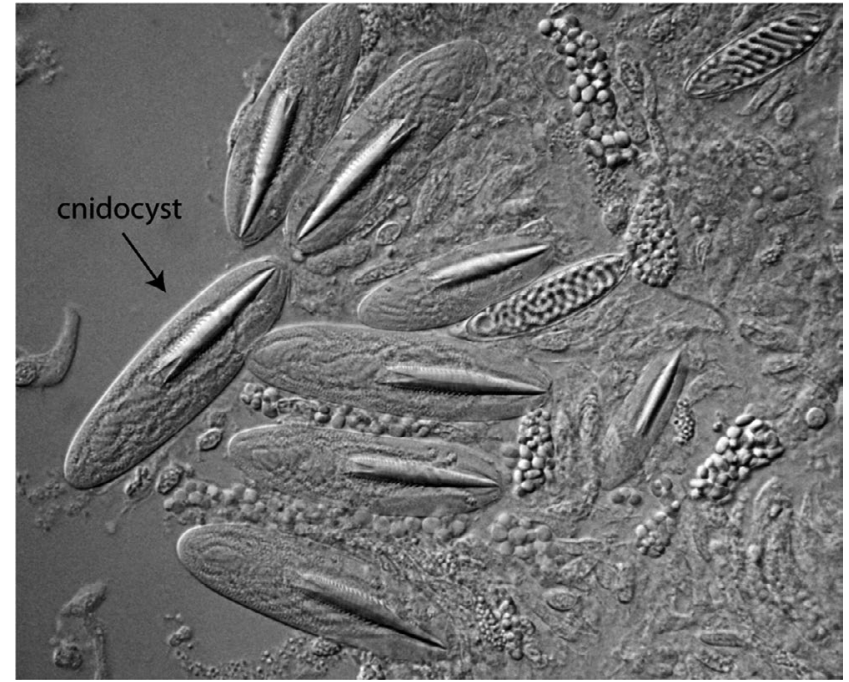

FIGURE 1 Undischarged cnidocysts from an anthozoan polyp

these light responses, so far we know that at least two of them are mediated by opsins: light-induced spawning in the hydrozoan jellyfish Clytia (Quiroga Artigas et al., 2018) and light modulation of cnidocyte discharge in Hydra (Plachetzki et al., 2012). In the jellyfish Clytia, a gonad-specific opsin of the xenopsin type (opsin9) controls secretion of a neuropeptide that causes oocyte maturation (Quiroga Artigas et al., 2018). Blue/cyan light induces the highest levels of oocyte maturation followed by gamete release, both of which fail to occur in genetically modified gonads that lack opsin9. In turn, another xenopsin (HmOps2) expressed in photosensory cells in the tentacles of Hydra polyps may modulate the discharge of neighboring stinging cells, the cnidocytes, in response to different intensities of blue light (Plachetzki et al., 2012). Here, the evidence for opsin is not via a knockout experiment, relying instead on a pharmacological agent that targeted a co-expressed ion channel known to be involved in opsin-based phototransduction.

Because cnidocytes were clearly present in ancestral cnidarians and benefit from strong sensory regulation, we hypothesize modulation of cnidocyte discharge by light was an ancestral function in cnidarians. A cnidocyte is a powerful weapon that produces a ballistic organelle, the cnidocyst, which is discharged upon proper cues (Figure 1; Kass-Simon et al., 2002). The cnidocyst itself is a capsule, very often containing toxins, with a harpoon-like tubule that releases its contents after the explosive firing. Cnidocytes are strongly regulated, because they are single-use and energetically costly to replace (Anderson \& Bouchard, 2009). Therefore, to maximize efficient use, multiple sensory modalities, including chemosensation, mechanosensation, and photosensation, regulate cnidocyte discharge, with cnidocytes in the tentacles being highly regulated for efficient prey capture (Anderson \& Bouchard, 2009). Assuming sensory regulation was always important for cnidocytes, then both function (regulation) and structure (cnidocyte) may date to the origin of cnidarians. In this study, we investigate whether this nonvisual light response occurs in distantly related groups of Cnidaria other than Hydra. Using wellestablished cnidocyte capture assays and phylogenetics, we test 
whether the intensity of blue light also affects the discharge of cnidocytes in four other eyeless species and whether this light response is likely to date to the cnidarian ancestor. Future studies could directly assess the molecular basis of the light modulated cnidocyte discharge. Our study brings into focus the early functional history of light responses in Cnidaria and how ancient sensory tasks may have facilitated eye origins by sustaining simple roles for extraocular photoreceptor cells.

\section{2 | MATERIALS AND METHODS}

\section{1 | Taxon sampling}

We tested how light conditions affect cnidocyte capture in four distantly related species, which represent four orders (Corallimorpharia, Actiniaria, Pennatulacea, Semaeostomeae), three subclasses (Hexacorallia, Octocorallia, Discomedusae), and two classes (Anthozoa, Scyphozoa). Most of these species occur in the coast of California and can be cultured over long periods of time, which helps with performing cnidocyte capture assays. We purposely chose distantly related species to span the breadth of Cnidaria, and we acknowledge that future studies that test more species and life stages could improve understanding of the evolution of cnidocyte firing.

\subsection{Animal cultures}

We cultured polyps of the sea anemone Diadumene lineata (Verrill, 1869) [=Haliplanella luciae] (Actiniaria, Hexacorallia) and the scyphozoan Aurelia aurita (Linnaeus, 1758) ("species 1" strain, Semaeostomeae, Discomedusae) in natural seawater at room temperature $\left(22^{\circ} \mathrm{C} \pm 1^{\circ} \mathrm{C}\right)$ under a $12: 12 \mathrm{hr}$ photoperiod with an artificial white fluorescent light. We also cultured specimens of the corallimorph Corynactis californica Carlgren, 1936 (Corallimorpharia, Hexacorallia), collected from oil platforms off Santa Barbara, California (USA) on 18 February 2015 and colonies of Renilla koellikeri Pfeffer, 1886 (Pennatulacea, Octocorallia), collected in the Santa Barbara Channel on 10 June 2015, in a seawater open system $\left(16^{\circ} \mathrm{C} \pm 2^{\circ} \mathrm{C}\right)$ with a $12: 12 \mathrm{hr}$ photoperiod. Animals were fed 3-day-old Selcon ${ }^{\circledR}$-enriched Artemia nauplii (San Francisco Strain Brine Shrimp Eggs) on a daily basis. We performed all experiments with animals starved for $24 \mathrm{hr}$.

\section{3 | Cnidocyte assays}

Because the polyp is widely accepted to be the ancestral stage among cnidarians, while the pelagic jellyfish evolved later in Medusozoa (Collins, 2002; Collins et al., 2006; Kayal et al., 2018), we focused our experiments on the polyp stage for inferring the ancestral state of the cnidocyte response to light in the cnidarian ancestor. All of our study species produce eyeless polyps, and only Aurelia produces a pelagic jellyfish (which possess simple eyes). Additionally, there are three types of cnidocytes (spirocytes, ptychocytes, and nematocytes) among anthozoans, but only the nematocytes are widely distributed across cnidarians. As such, when we refer to cnidocytes throughout the text, we are specifically referring to nematocytes.

Cnidocyte capture assays followed the method described in Watson and Hessinger (1989). After double-coating fishing line with $20 \%(\mathrm{w} / \mathrm{v})$ gelatin preheated to $\sim 70^{\circ} \mathrm{C}, 2 \mathrm{~cm}$-long monofilament fishing line probes (Essentials South Bend $\AA$ ) were left to dry for $\sim 20 \mathrm{~min}$ and then used for contacting one tentacle of each individual. We exposed healthy individuals to one of two different light intensities (dim light, $0.1 \mathrm{~W} / \mathrm{cm}^{2}$; bright light, $2.8 \mathrm{~W} / \mathrm{cm}^{2}$ ) from a blue LED (SuperBright LEDs) light source with a spectral peak at $470 \mathrm{~nm}$ for approximately two (Aurelia, $N=33$ ), three (Corynactis, $N=30$; Renilla, bright light, $N=39$; dim light, $N=27$; maintained at $\sim 16^{\circ} \mathrm{C}$ in a cold chamber during experimentation), or four hours (Diadumene, dim light, $N=40$; bright light, $N=33$ ). Because polyps took different amounts of time to relax after being moved into the experimental setup, they were exposed for varying amounts of time. Light intensity was measured using the Jaz spectrometer (Ocean Optics). Gelatin-coated probes were mounted in $100 \%$ glycerol, and discharged nematocysts were counted at $400 \times$ or $600 \times$ magnification of an Olympus BX61 microscope. We counted nematocysts by searching the whole length and width of the probe (one probe per individual) with proper focal adjustments. Probes were discarded whenever counting could not be done by the lack of a focal point or agglomeration of nematocysts.

\section{4 | Phylogenetic analysis}

We used a maximum likelihood approach to infer the ancestral states (light modulated cnidocyte discharge, present or absent) on the time-calibrated phylogeny from Picciani et al. (2018). We used R 4.0.2 and the function rayDISC from the R package corHMM v1.22 (Beaulieu et al., 2013) to estimate the marginal likelihoods of internal nodes with symmetrical rates model since the asymmetrical one was not significantly better and could lead to overparameterization (likelihood ratio test; chi-square test; $d f=1 ; p=0.1$ ). Because genetic data are scarce for Renilla, this species is missing from the phylogeny, and thus, we scored another pennatulacean (Umbellula) in our tree as a surrogate taxa. Additionally, because outgroups lack cnidocytes altogether, we used a root prior to fix the root state as absent.

\section{5 | Statistical analysis and accessibility}

We analyzed counts of nematocysts captured in the gelatin probes using R 3.6.1. For every species, data were non-normal (ShapiroWilk test, $p<0.001$; except for Diadumene, which had data from treatment with dim light following a normal distribution) and frequency distributions were highly skewed though they had roughly the same shape. Given that, we used the Wilcoxon rank-sum test to 
compare sample means of each light treatment, assuming a significance level $(\alpha)$ of 0.05. Data for two species, Renilla and Corynactis, possessed many trials when no cnidocytes fired. To account for such heavily skewed data, we also used a zero-inflated negative binomial regression (R package "pscl") to test whether experimental condition (bright vs. dim light) and species (the four tested, mentioned above) could explain variation in cnidocyte firing counts beyond a statistically distinct process generating the accumulation zeros in our data.

\section{3 | RESULTS}

\section{1 | Light modulates cnidocyte discharge in distantly related cnidarians}

Our analyses reveal a clear trend across distantly related cnidarians to use light for modulating the discharge of their cnidocytes
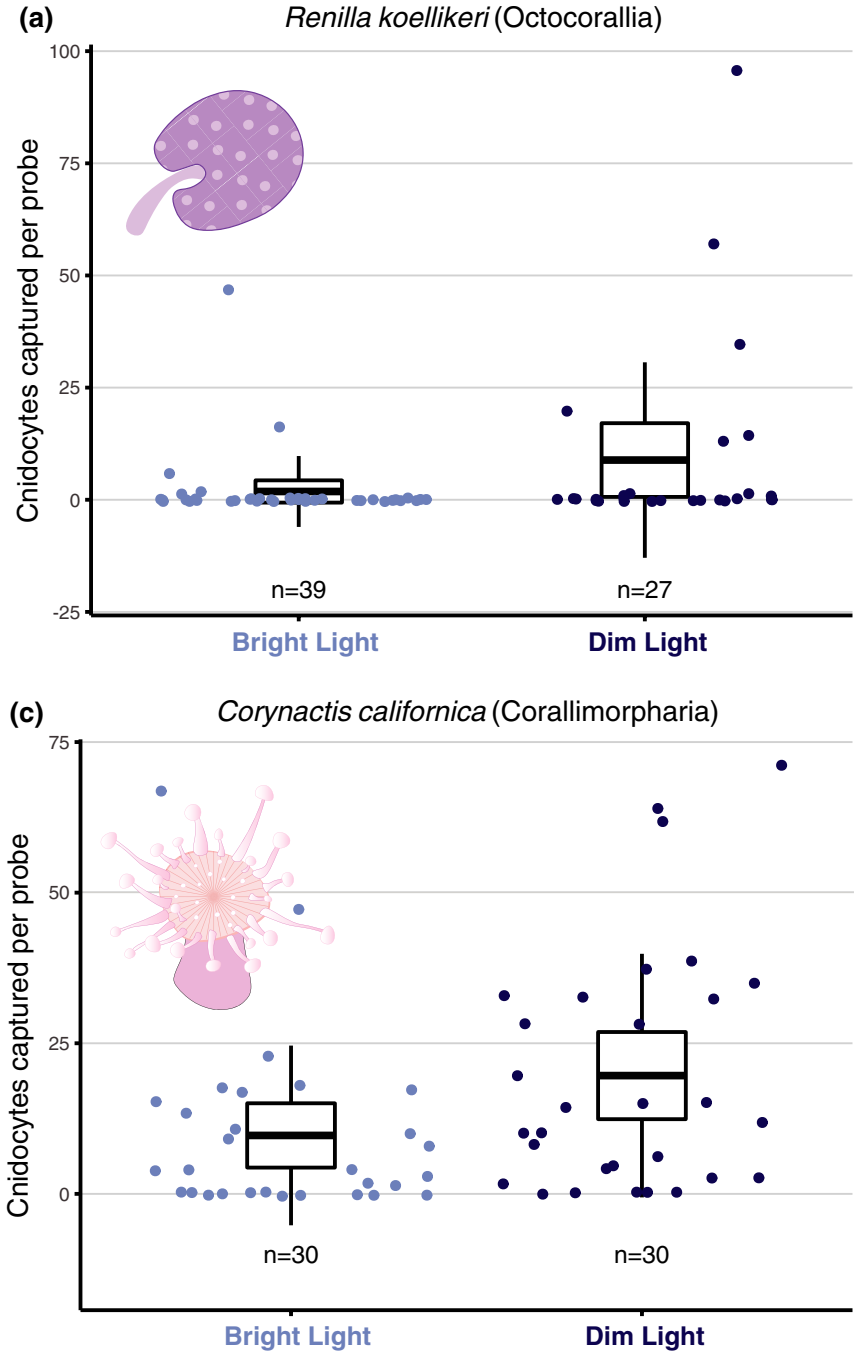

(Figure 2) and indicate the cnidarian ancestor was also able to do so (Figure 3). Overall, the discharge of cnidocytes into probes was significantly higher for polyps exposed to a dim compared with bright blue LED light (Figure 2). Our statistical power was very high ( 100\%) for Diadumene and Aurelia, indicating that we can be very confident in the effect of light intensity on cnidocyte discharge in these two long-diverged taxa ( 700 mya). Conversely, power was lower for the other two taxa (Renilla and Corynactis; $40.3 \%$ and $52.9 \%$, respectively) so that despite significant effects ( $p=0.025$ in Corynactis; $p=0.022$ in Renilla), these should be considered with caution because low power may increase the chance of false positive results (Christley, 2010).

Our zero-inflated negative binomial model agrees with our other statistical tests and additionally finds evidence for an overabundance of zeros in our count data, especially among dim conditions and within Corynactis and Renilla (Zero-inflation model, $p<0.01$ for each). This model was preferred to a simple negative binomial (b) Diadumene lineata (Actiniaria)

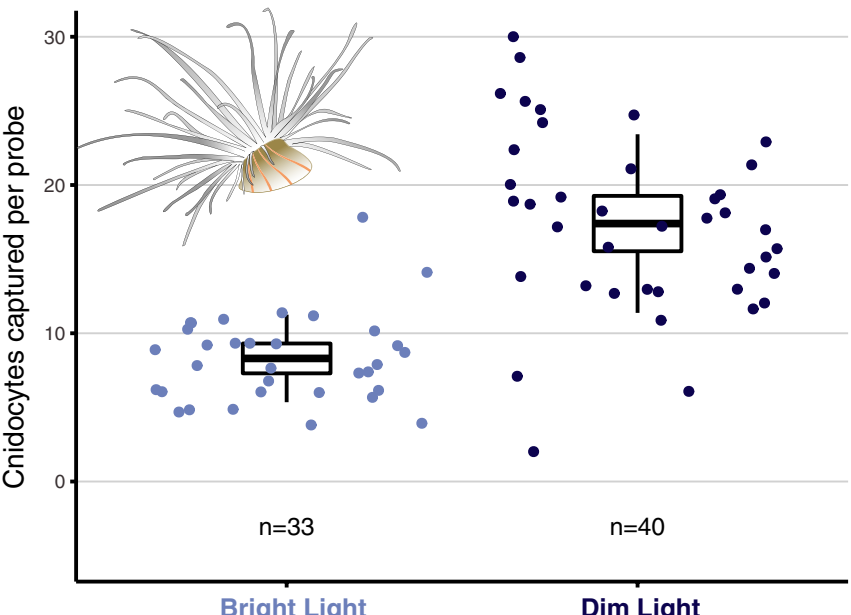

(d)

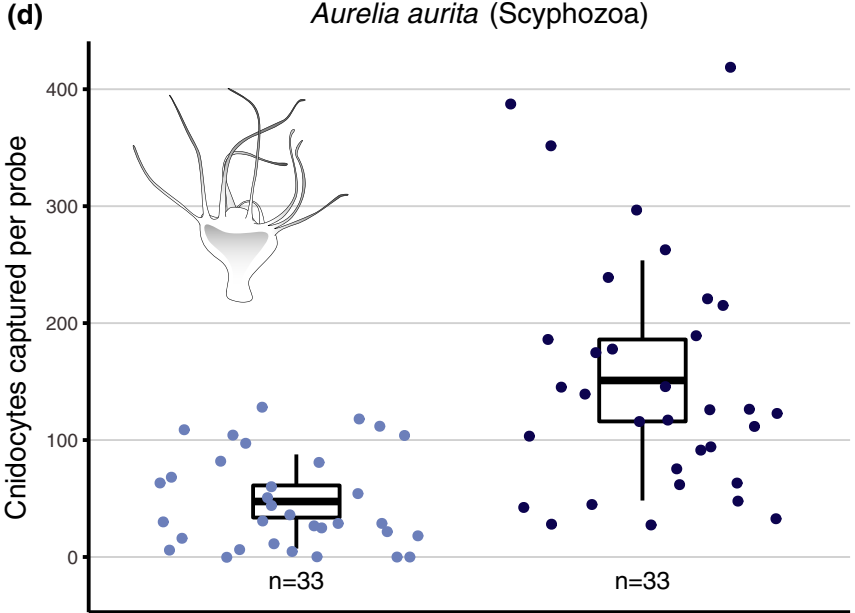

Bright Light

Dim Light

FIGURE 2 Cnidocyte discharge increases when polyps are exposed to dim blue light, a response conserved across long-diverged cnidarian species. Under dim blue light $\left(470 \mathrm{~nm} ; 0.1 \mathrm{~W} / \mathrm{cm}^{2}\right)$, discharge of cnidocysts in the gelatin matrix was significantly higher than in bright blue light $\left(470 \mathrm{~nm} ; 2.8 \mathrm{~W} / \mathrm{cm}^{2}\right.$ ) assays (Wilcoxon Rank-Sum Test, two-tailed; Aurelia: $p<0.0001$, Corynactis: $p=0.025$, Diadumene: $p<0.0001$, Renilla: $p=0.022$; see Materials and Methods for details). Center lines in box plots correspond to the sample mean, top and bottom extremes represent upper and lower $95 \%$ confidence interval points, and whiskers are one standard deviation lines 


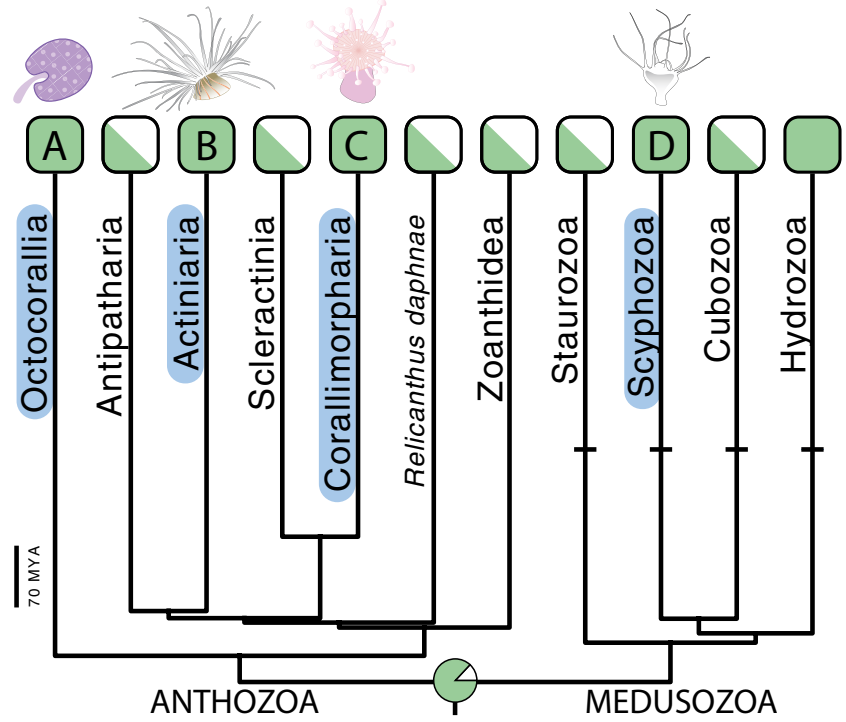

FIGURE 3 Maximum likelihood ancestral state reconstruction on the main phylogeny from Picciani et al. (2018). Marginal likelihoods of ancestral states (light modulated cnidocyte discharge present, green; absent, white) at the cnidarian ancestor node are shown in the pie chart and inferred with a symmetric Markov twostate model (equal rates) of trait evolution. Letters and blue ovals show where studied species are placed in the phylogeny (A: Renilla, B: Diadumene, C: Corynactis, D: Aurelia). Tip states of groups for which we lack information on light modulated cnidocyte discharge are scored as missing data and shown as rectangles half colored in green. Horizontal bars indicate lineages in which eyes convergently evolved. Scale bar denotes time in millions of years. See Figure S1 for the whole phylogeny with ancestral states

regression (Vuong test statistic for AIC values, $p<0.001$ ), indicating that accounting for excess zeros in our cnidocyte firing is a better descriptor of our data than not. In this model, both species and experimental condition significantly explain variation in cnidocyte firing (Analysis of Deviance, $p<0.01$ for each). Taken together, and as is most evident in distribution of our data (Figure 2), we believe that light modulation of cnidocyte firing is likely across these taxa.

\section{2 | Species-specific variation in numbers of discharged cnidocytes}

The octocoral Renilla discharged substantially fewer cnidocytes on average (from each treatment) than all other species, while the scyphopolyp Aurelia discharged more cnidocytes than the octocoral, the sea anemone Diadumene and the corallimorph Corynactis. That could be explained by either a comparable density of cnidocytes among species but differential use, variation of cnidocyte density in tentacles among species or a combination of both. For instance, octocorals often lack cnidocytes altogether, and other species of Renilla possess low numbers of a simple type of nematocyte (Mariscal \& Bigger, 1977). In turn, scyphopolyps depend primarily on only one type of cnidocyte, the nematocyte, as opposed to most anthozoans, which use two types of cnidocytes (nematocytes and spirocytes) for lassoing prey (Fautin, 2009). That the scyphopolyp Aurelia relies only on nematocytes might explain its higher discharge averages compared with other species.

\section{4 | DISCUSSION}

Our study presents empirical support for a sensory task that we suggest as a possible role for ancestral photoreceptors that predate cnidarian eyes. By testing whether the modulation of cnidocyte discharge by light occurs among long-diverged cnidarian lineages and reconstructing the state of the cnidarian ancestor, we find support for the hypothesis that this light response is a deeply conserved sensory task preserved over millions of years. Because we find a broad diversity of cnidarian polyps discharge significantly more cnidocytes during exposure to dim blue light compared with bright blue light, we suggest that ancestral photoreceptors in Cnidaria regulated the discharge of cnidocytes. Several ecological reasons could explain why distantly related species tend to discharge more cnidocytes in dimmer light (see discussion in Plachetzki et al. 2012). For example, fine tuning cnidocyte discharge to maximize prey capture either at dusk, when zooplankton migrate to surface waters, or when prey items cast a shadow on the polyp. Although we do not rule out other possible light sensing functions, because cnidocyte discharge is still the primary means of defense and prey capture of almost all cnidarians, such a long-standing photoreceptive function could have facilitated multiple convergent eye origins in the group by maintaining phototransduction pathways and enabling them to be later exploited for vision.

Organization of cnidocytes and their sensory apparatus vary extensively between cnidarian classes (Anderson \& Bouchard, 2009), yet a similar innervation pattern (Anderson et al., 2004) suggests photoreceptor cells could still have persisted in the circuitry controlling cnidocyte discharge. Spatial positioning of cnidocytes in tentacles varies considerably-from patchy in hydrozoans and scyphozoans to uniform in sea anemones and corals (Anderson \& Bouchard, 2009). Additionally, receptor complexes associated with cnidocytes can be produced solely by the cnidocytes themselves or receive projections from nearby ciliary cells (Watson \& Mire-Thibodeaux, 1994). Given such seemingly divergent organization, an alternative to homology of light modulation of cnidocyte discharge would be convergence of such light responsiveness via repeated co-option of photoreceptor cells into cnidocyte circuitry. If convergent, the ancestral cnidocyte circuitry would have lacked photoreceptor cells, which would have been later independently assimilated into the circuitry of cnidocytes. But cnidarian photoreceptor cells are strongly peptidergic (Martin, 2002, 2004; Plickert \& Schneider, 2004), and cnidocytes are innervated by networks of peptidergic neurons in all cnidarian classes regardless of their cnidocyte organization (Anderson et al., 2004; Westfall, 2004). These observations on peptidergic neurons, coupled with our inference that light modulation of cnidocyte discharge was ancestral, are consistent with a hypothesis that the cnidarian ancestor possessed photoreceptor cells that could 
send modulatory signals to cnidocytes and that these cells likely persisted in cnidocyte circuitry over evolutionary time.

Of the various light sensing genes in cnidarians, only xenopsins (called cnidops in cnidarians) are known to mediate photoreception in both Medusozoa and eyeless Anthozoa, suggesting that xenopsins could be used to sense light for cnidocyte discharge. For instance, different light sensing molecules, either nonopsin proteins or opsin types other than xenopsin, could be used for light detection in species of anthozoans. Even though anthozoans can sense light with cryptochromes and two opsin types besides xenopsin (Gornik et al., no date; Ramirez et al., 2016; Reitzel et al., 2013; Picciani et al., 2018), only the xenopsin seems to be used for light sensing by medusozoans. Interestingly, it is both the light sensitive molecule in photoreceptor cells of eyes and photosensory neurons that modulate the discharge of cnidocytes in Hydra (Plachetzki et al., 2012). It is likely that a homologous light response would be mediated by a light sensing molecule shared among all cnidarians, such as xenopsin. Demonstrating that the modulation of cnidocyte discharge in anthozoans is done with photoreceptors that use xenopsins would reinforce photoreceptor homology.

Other roles besides modulation of cnidocyte discharge are also possible for photoreceptors in the cnidarian ancestor, thought to be a solitary polyp lacking symbionts (Kayal et al., 2018). Several functions, including larval settlement and phototaxis, could also be ancestral-but we do not yet know whether they use opsins. If not opsin-mediated, it seems unlikely such photoreceptors became assimilated into eyes that invariably use opsin. A topic for future research would be to test whether other light-dependent functions are mediated by opsins, and if so, whether the functions are ancestral in Cnidaria. Second, opsin-expressing ectodermal cells in the gonads of Clytia control oocyte maturation (Quiroga Artigas et al., 2018), so that spawning is another candidate for an ancestral photoreceptive function in cnidarians. Testing whether light-influenced spawning is ancestral would require a survey of other species besides Clytia. A broad survey could be facilitated by the many available reports of light-influenced spawning in Cnidaria (see item S1 in Picciani et al., 2018). Understanding the phototransduction pathways underlying spawning across species using genetic and experimental approaches would also be important to uncover the identity of photoreceptor cells and their relationship to eye precursors.

In addition to photoreceptor cells, other key precursor modules like pigments and crystallins probably predated cnidarian eye origins and served other organismal functions prior to visual function. For instance, one module-the biosynthesis machinery of melanin that includes tyrosinases-is present in species of both Anthozoa and Medusozoa (Dunlap et al., 2013; Esposito et al., 2012), the two major cnidarian sister lineages, and therefore could also be ancestral. Melanin synthesis is involved in many biological processes outside of cnidarian eyes, including functioning as a trigger for scyphopolyps to strobilate and produce jellyfish (Berking et al., 2005; Van den Branden et al., 1980; Van den Branden, Van den Sande, \& Decleir, 1980). Moreover, melanin is also used by corals, sea fans, and anemones to create a physical barrier against pathogens, and melanin synthesis is correlated with disease resistance in corals (Mydlarz et al., 2008; Mydlarz \& Palmer, 2011; Palmer et al., 2008, 2012; Petes et al., 2003; Zaragoza et al., 2014). Another precursor module, the crystallin proteins, forms lenses in the eyes of box jellyfish and may be derived from proteins with nonoptical functions (Piatigorsky et al., 1989, 2001; Piatigorsky et al., 1993). We know relatively little about the origins, both structural and functional, of box jellyfish lens crystallins, though they are thought to be closely related to vertebrate saposins (Piatigorsky et al., 2001). Crystallin homologs seem to occur in sea anemones (Nicosia et al., 2014) and could perhaps be present in other lineages of eyeless cnidarians or could have occurred ancestrally and been lost in most eyeless species.

By testing a wide breadth of cnidarian diversity for a lightinfluenced response known to involve a family of opsins used for vision, our results highlight one possible early role for eye precursors in Cnidaria was to modulate cnidocyte discharge. These results contribute to our understanding of eye evolution by using a phylogenetic context to propose an explanation for where the photoreceptor cells of eyes come from, and what functions they possibly had before becoming functionally integrated with other structures to mediate vision. It also raises interesting questions about how sensory tasks continued to evolve in lineages that acquired eyes. Which novel functions were cnidarians able to perform once they evolved directional photoreceptors and image-forming eyes? Did those new functions supersede ancestral functions? As proposed by Nilsson (2013), the evolution of increasingly complex visual tasks can be studied concomitantly with eye morphology so we can understand evolutionary trajectories accompanying both function and structure. By advancing a possible ancient role for cnidarian eye precursors, our study helps to start dissecting the functional drivers that can elaborate morphological complexity.

\section{ACKNOWLEDGMENTS}

We thank Professor Glen Watson for the donation of polyps of Diadumene (=Haliplanella) lineata, Jan Bielecki and Natalie Colburn for help with animal culture and data collection. We used computational facilities administered by the Center for Scientific Computing at the CNSI and MRL (NSF MRSEC; DMR-1720256) and purchased through NSF CNS-1725797, with incredible assistance from Fuzzy Rogers and Paul Weakliem. Two anonymous reviewers helped improve the manuscript with thoughtful suggestions. This study was supported by CAPES (Coordenação de Aperfeiçoamento de Pessoal de Nível Superior) through a doctoral fellowship to NP (process BEX13130-13/7) in the program Science without Borders and through a research grant from NSF to THO (IOS-1754770).

\section{CONFLICT OF INTEREST}

The authors declare no conflicts of interest.

\section{AUTHOR CONTRIBUTIONS}

Natasha Picciani: Conceptualization (equal); Data curation (lead); Formal analysis (equal); Funding acquisition (equal); Investigation 
(equal); Methodology (equal); Visualization (equal); Writing-original draft (lead); Writing-review \& editing (equal). Jamie R. Kerlin: Investigation (equal); Writing-review \& editing (equal). Katia Jindrich: Investigation (equal); Writing-review \& editing (equal). Nicholai M. Hensley: Formal analysis (equal); Visualization (equal); Writingreview \& editing (equal). David A. Gold: Investigation (equal); Writing-review \& editing (equal). Todd H. Oakley: Conceptualization (equal); Funding acquisition (equal); Methodology (equal); Resources (lead); Writing-original draft (supporting); Writing-review \& editing (equal).

\section{ETHICAL APPROVAL}

The authors followed all guidelines for ethical treatment of the animals.

\section{OPEN RESEARCH BADGES}

\section{(11)}

This article has earned an Open Data Badge for making publicly available the digitally-shareable data necessary to reproduce the reported results. The data is available at https://doi.org/10.5061/ dryad.9w0vt4bds.

\section{DATA AVAILABILITY STATEMENT}

Raw datasets and analysis code are deposited in the Dryad repository https://doi.org/10.5061/dryad.9w0vt4bds.

\section{ORCID}

Natasha Picciani iD https://orcid.org/0000-0001-9615-5020 Jamie R. Kerlin (iD https://orcid.org/0000-0001-8158-6231 Katia Jindrich (iD https://orcid.org/0000-0001-8801-325X Nicholai M. Hensley (iD https://orcid.org/0000-0003-0681-0808 David A. Gold iD https://orcid.org/0000-0003-0135-4022 Todd H. Oakley iD https://orcid.org/0000-0002-4478-915X

\section{REFERENCES}

Anderson, P. A. V., \& Bouchard, C. (2009). The regulation of cnidocyte discharge. Toxicon: Official Journal of the International Society on Toxinology, 54(8), 1046-1053. https://doi.org/10.1016/j.toxic on.2009.02.023

Anderson, P. A. V., Thompson, L. F., \& Moneypenny, C. G. (2004). Evidence for a common pattern of peptidergic innervation of cnidocytes. The Biological Bulletin, 207, 141-146. https://doi.org/10.2307/1543588

Beaulieu, J. M., O'Meara, B. C., \& Donoghue, M. J. (2013). Identifying hidden rate changes in the evolution of a binary morphological character: The evolution of plant habit in campanulid angiosperms. Systematic Biology, 62(5), 725-737. https://doi.org/10.1093/sysbio/ syt034

Berking, S., Czech, N., Gerharz, M., Herrmann, K., Hoffmann, U., Raifer, H., Sekul, G., Siefker, B., Sommerei, A., \& Vedder, F. (2005). A newly discovered oxidant defence system and its involvement in the development of Aurelia aurita (Scyphozoa, Cnidaria): Reactive oxygen species and elemental iodine control medusa formation. The International Journal of Developmental Biology, 49(8), 969-976. https://doi.org/10.1387/ijdb.052024sb
Boch, C. A., Ananthasubramaniam, B., Sweeney, A. M., Doyle, F. J., \& Morse, D. E. (2011). Effects of light dynamics on coral spawning synchrony. The Biological Bulletin, 220(3), 161-173. https://doi. org/10.1086/BBLv220n3p161

Christley, R. M. (2010). Power and error: Increased risk of false positive results in underpowered studies. The Open Epidemiology Journal, 3(1), 16-19. https://doi.org/10.2174/1874297101003010016

Collins, A. G. (2002). Phylogeny of Medusozoa and the evolution of cnidarian life cycles. Journal of Evolutionary Biology, 418-432, https:// doi.org/10.1046/j.1420-9101.2002.00403.x

Collins, A. G., Schuchert, P., Marques, A. C., Jankowski, T., Medina, M., \& Schierwater, B. (2006). Medusozoan phylogeny and character evolution clarified by new large and small subunit rDNA data and an assessment of the utility of phylogenetic mixture models. Systematic Biology, 55(1), 97-115. https://doi.org/10.1080/1063515050 0433615

Dunlap, W. C., Starcevic, A., Baranasic, D., Diminic, J., Zucko, J., Gacesa, R., van Oppen, M. J. H., Hranueli, D., Cullum, J., \& Long, P. F. (2013). KEGG orthology-based annotation of the predicted proteome of Acropora digitifera: ZoophyteBase - An open access and searchable database of a coral genome. BMC Genomics, 14, 509. https://doi. org/10.1186/1471-2164-14-509

Esposito, R., D'Aniello, S., Squarzoni, P., Pezzotti, M. R., Ristoratore, F., \& Spagnuolo, A. (2012). New insights into the evolution of metazoan tyrosinase gene family. PLoS One, 7(4), e35731. https://doi. org/10.1371/journal.pone.0035731

Gorbunov, M. Y., \& Falkowski, P. G. (2002). Photoreceptors in the cnidarian hosts allow symbiotic corals to sense blue moonlight. Limnology and Oceanography, 47(1), 309-315. https://doi.org/10.4319/ lo.2002.47.1.0309

Gornik, S. G., Bergheim, B. G., Morel, B., Stamatakis, A., Foulkes, N. S., \& Guse, A. (2020). Photoreceptor diversification accompanies the evolution of anthozoa. Molecular Biology and Evolution, http://dx.doi. org/10.1093/molbev/msaa304

Kass-Simon, G., \& Scappaticci, Jr., A. A. (2002). The behavioral and developmental physiology of nematocysts. Canadian Journal of Zoology, 80(10), 1772-1794. https://doi.org/10.1139/z02-135

Kayal, E., Bentlage, B., Sabrina Pankey, M., Ohdera, A. H., Medina, M., Plachetzki, D. C., Collins, A. G., \& Ryan, J. F. (2018). Phylogenomics provides a robust topology of the major cnidarian lineages and insights on the origins of key organismal traits. BMC Evolutionary Biology, 18(1), 68. https://doi.org/10.1186/s12862-018-1142-0

Leung, N. Y., \& Montell, C. (2017). Unconventional roles of opsins. Annual Review of Cell and Developmental Biology, 33(1), 241-264. https://doi. org/10.1146/annurev-cellbio-100616-060432

Martin, V. J. (2002). Photoreceptors of cnidarians. Canadian Journal of Zoology, 80(10), 1703-1722. https://doi.org/10.1139/z02-136

Martin, V. J. (2004). Photoreceptors of cubozoan jellyfish. Hydrobiologia, 530(531), 135-144. https://doi.org/10.1007/s10750-004-2674-4

Mason, B., Schmale, M., Gibbs, P., Miller, M. W., Wang, Q., Levay, K., Shestopalov, V., \& Slepak, V. Z. (2012). Evidence for multiple phototransduction pathways in a reef-building coral. PLoS One, 7(12), 19. https://doi.org/10.1371/journal.pone.0050371

Miller, R. L. (1979). Sperm chemotaxis in the hydromedusae. I. Speciesspecificity and sperm behavior. Marine Biology, 53(2), 99-113. https:// doi.org/10.1007/bf00389182

Miranda, L.S., \& Collins A. G. (2019). Eyes in Staurozoa (Cnidaria): a review. PeerJ, 7, e6693. http://dx.doi.org/10.7717/peerj.6693

Mydlarz, L. D., Holthouse, S. F., Peters, E. C., \& Harvell, C. D. (2008). Cellular responses in sea fan corals: Granular amoebocytes react to pathogen and climate stressors. PLoS One, 3(3), e1811. https://doi. org/10.1371/journal.pone.0001811

Mydlarz, L. D., \& Palmer, C. V. (2011). The presence of multiple phenoloxidases in Caribbean reef-building corals. Comparative Biochemistry 
and Physiology - A Molecular and Integrative Physiology, 159(4), 372378. https://doi.org/10.1016/j.cbpa.2011.03.029

Nicosia, A., Maggio, T., Mazzola, S., Gianguzza, F., Cuttitta, A., \& Costa, S. (2014). Characterization of small HSPs from Anemonia viridis reveals insights into molecular evolution of alpha crystallin genes among cnidarians. PLoS One, 9, e105908. https://doi.org/10.1371/journ al.pone.0105908

Nilsson, D.-E. (2009). The evolution of eyes and visually guided behaviour. Philosophical Transactions of the Royal Society of London. Series B, Biological Sciences, 364(1531), 2833-2847. https://doi. org/10.1098/rstb.2009.0083

Nilsson, D.-E. (2013). Eye evolution and its functional basis. Visual Neuroscience, 30, 5. https://doi.org/10.1017/S0952523813000035

Oakley, T. H., \& Speiser, D. I. (2015). How complexity originates: The evolution of animal eyes. Annual Review of Ecology, Evolution, and Systematics, 46(1), 237-260. https://doi.org/10.1146/annurev-ecols ys-110512-135907

Palmer, C. V., Bythell, J. C., \& Willis, B. L. (2012). Enzyme activity demonstrates multiple pathways of innate immunity in Indo-Pacific anthozoans. Proceedings of the Royal Society B: Biological Sciences, 279(1743), 3879-3887.

Palmer, C. V., Mydlarz, L. D., \& Willis, B. L. (2008). Evidence of an inflammatory-like response in non-normally pigmented tissues of two scleractinian corals. Proceedings of the Royal Society B: Biological Sciences, 275(1652), 2687-2693.

Petes, L. E., Harvell, C. D., Peters, E. C., Webb, M., \& Mullen, K. M. (2003). Pathogens compromise reproduction and induce melanization in Caribbean Sea fans. Marine Ecology Progress Series, 264, 167-171. https://doi.org/10.3354/meps264167

Piatigorsky, J., Horwitz, J., Kuwabara, T., \& Cutress, C. E. (1989). The cellular eye lens and crystallins of cubomedusan jellyfish. Journal of Comparative Physiology A, 164(5), 577-587. https://doi.org/10.1007/ BF00614500

Piatigorsky, J., Horwitz, J., \& Norman, B. L. (1993). J1-crystallins of the cubomedusan jellyfish lens constitute a novel family encoded in at least three intronless genes. The Journal of Biological Chemistry, 268(16), 11894-11901. https://doi.org/10.1016/S0021 $-9258(19) 50284-8$

Piatigorsky, J., Norman, B., Dishaw, L. J., Kos, L., Horwitz, J., Steinbach, P. J., \& Kozmik, Z. (2001). J3-crystallin of the jellyfish lens: Similarity to saposins. Proceedings of the National Academy of Sciences of the United States of America, 98(22), 12362-12367.

Picciani, N., Kerlin, J. R., Sierra, N., Swafford, A. J. M., Ramirez, M. D., Roberts, N. G., Cannon, J. T., Daly, M., \& Oakley, T. H. (2018). Prolific origination of eyes in Cnidaria with co-option of non-visual opsins. Current Biology, 28(15), 2413-2419.e4. https://doi.org/10.1016/j. cub.2018.05.055

Plachetzki, D. C., Fong, C. R., \& Oakley, T. H. (2012). Cnidocyte discharge is regulated by light and opsin-mediated phototransduction. BMC Biology, 10, 17. https://doi.org/10.1186/1741-7007-10-17

Plickert, G., \& Schneider, B. (2004). Neuropeptides and photic behavior in Cnidaria. Hydrobiologia, 530(531), 49-57. https://doi.org/10.1007/ s10750-004-2689-x

Quiroga Artigas, G., Lapébie, P., Leclère, L., Takeda, N., Deguchi, R., Jékely, G., Momose, T., Houliston, E. (2018). A gonad-expressed opsin mediates light-induced spawning in the jellyfish. eLife, 7, e29555. https:// doi.org/10.7554/eLife.29555
Ramirez, M. D., Pairett, A. N., Pankey, M. S., Serb, J. M., Speiser, D. I., Swafford, A. J., \& Oakley, T. H. (2016). The last common ancestor of most bilaterian animals possessed at least nine opsins. Genome Biology and Evolution, 8(12), 3640-3652.

Ramirez, M. D., Speiser, D. I., Sabrina pankey, M., \& Oakley, T. H. (2011). Understanding the dermal light sense in the context of integrative photoreceptor cell biology. Visual Neuroscience, 28(4), 265-279. https://doi.org/10.1017/S0952523811000150

Reitzel, A. M., Tarrant, A. M., \& Levy, O. (2013). Circadian clocks in the cnidaria: Environmental entrainment, molecular regulation, and organismal outputs. Integrative and Comparative Biology, 53(1), 118130. https://doi.org/10.1093/icb/ict024

Sawyer, S. J., Dowse, H. B., \& Shick, J. M. (1994). Neurophysiological correlates of the behavioral response to light in the sea anemone Anthopleura elegantissima. The Biological Bulletin, 186(2), 195-201.

Schuyler, Q., \& Sullivan, B. K. (1997). Light responses and die1 migration of the scyphomedusa Chrysaora quinquecirrha in mesocosms. Journal of Plankton Research. Retrieved from https://academic.oup.com/ plankt/article-abstract/19/10/1417/1488708

Swafford, A. J. M., \& Oakley, T. H. (2019). Light-induced stress as a primary evolutionary driver of eye origins. Integrative and Comparative Biology, 59(4), 739-750. http://dx.doi.org/10.1093/icb/icz064

Van den Branden, C., Van den Sande, P., \& Decleir, W. (1980). A study of pigments in some developmental stages of Aurelia aurita Lam. Acta Zoologica et Pathologica Antverpiensia, 75, 19-28.

Van den Branden, C., Van de Mieroop, A., Anthoons, J., Decleir, W. (1980). An histochemical study of pigment in strobilae and ephyrae of Aurelia aurita Lam. Biologisch Jaarboek Dodonaea, 48, 149-152.

Watson, G. M., \& Hessinger, D. A. (1989). Cnidocytes and adjacent supporting cells form receptor-effector complexes in anemone tentacles. Tissue \& Cell, 21(1), 17-24. https://doi. org/10.1016/0040-8166(89)90017-7

Watson, G. M., \& Mire-Thibodeaux, P. (1994). The cell biology of nematocysts. International Review of Cytology, 156, 275-300.

Westfall, J. A. (2004). Neural pathways and innervation of cnidocytes in tentacles of sea anemones. Hydrobiologia, 530-531(1-3), 117-121. http://dx.doi.org/10.1007/s10750-004-2678-0

Zaragoza, W. J., Krediet, C. J., Meyer, J. L., Canas, G., Ritchie, K. B., \& Teplitski, M. (2014). Outcomes of infections of sea anemone Aiptasia pallida with Vibrio spp. pathogenic to corals. Microbial Ecology, 68(2), 388-396. https://doi.org/10.1007/s00248-014-0397-2

\section{SUPPORTING INFORMATION}

Additional supporting information may be found online in the Supporting Information section.

How to cite this article: Picciani N, Kerlin JR, Jindrich K, Hensley NM, Gold DA, Oakley TH. Light modulated cnidocyte discharge predates the origins of eyes in Cnidaria. Ecol Evol. 2021;11:3933-3940. https://doi.org/10.1002/ece3.7280 